Article

\title{
Investigation of Outbreaks of Extended-Spectrum Beta-Lactamase-Producing Klebsiella Pneumoniae in Three Neonatal Intensive Care Units Using Whole Genome Sequencing
}

\author{
Sammy Frenk ${ }^{1, *}$, Nadya Rakovitsky ${ }^{1}$, Elizabeth Temkin ${ }^{1}$, Vered Schechner ${ }^{2,3}$, \\ Regev Cohen ${ }^{4,5}{ }^{(}$, Bat Sheva Kloyzner ${ }^{6}$, Mitchell J. Schwaber ${ }^{1,3}$, Ester Solter ${ }^{1}$, \\ Shoshana Cohen ${ }^{4}$, Sarit Stepansky ${ }^{2}$ and Yehuda Carmeli ${ }^{1,3}$ \\ 1 National Institute for Antibiotic Resistance and Infection Control, Ministry of Health, Tel Aviv 64239, Israel; \\ nadyarak@tlvmc.gov.il (N.R.); lizt@tlvmc.gov.il (E.T.); mitchells@tlvmc.gov.il (M.J.S.); \\ esterso@tlvmc.gov.il (E.S.); yehudac@tlvmc.gov.il (Y.C.) \\ 2 Division of Epidemiology and Preventive Medicine, Tel Aviv Sourasky Medical Center, Tel Aviv 64239, Israel; \\ vereds@tlvmc.gov.il (V.S.); saritst@tlvmc.gov.il (S.S.) \\ 3 Sackler Faculty of Medicine, Tel Aviv University, Tel Aviv 69978, Israel \\ 4 Infectious Diseases Unit, Laniado Hospital, Netanya 42150, Israel; regevc@laniado.org.il (R.C.); \\ shoshic@laniado.org.il (S.C.) \\ 5 Ruth and Bruce Rappaport Faculty of Medicine, Technion-Israel Institute of Technology, Haifa 3525433, Israel \\ 6 Department of Epidemiology, Mayanei Hayeshua Medical Center, Bnei Brak 51544, Israel; kloyz@mhmc.co.il \\ * Correspondence: sammyf@tlvmc.gov.il; Tel.: +972-3-6974892
}

Received: 1 September 2020; Accepted: 14 October 2020; Published: 16 October 2020

check for updates

\begin{abstract}
Infections caused by extended-spectrum beta-lactamase-producing Klebsiella pneumoniae (ESBL-KP) are on a constant rise and are a noted cause of outbreaks in neonatal intensive care units (NICUs). We used whole genome sequencing (WGS) to investigate the epidemiology of consecutive and overlapping outbreaks caused by ESBL-KP in NICUs in three hospitals in close proximity. Clonality of 43 ESBL-KP isolates from 40 patients was determined by BOX-PCR. Short-read sequencing was performed on representative isolates from each clone. The dominant clones from each NICU were sequenced using long-read sequencing. Bioinformatics methods were used to define multilocus sequence type (MLST), analyze plasmid content, resistomes, and virulence factors. In each NICU, we found a unique dominant clone (ST985, ST37, and ST35), each belonging to a distinct sequence type (ST), as well as satellite clones. A satellite strain in NICU-2 (ST35) was the dominant strain in NICU-3, where it was isolated four weeks later, suggesting transmission. NICU-1- and

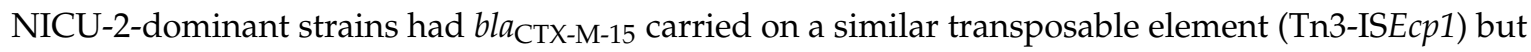
at different locations: on a plasmid and on the chromosome, respectively. We concluded that the overlapping ESBL-KP outbreaks were a combination of clonal transmission within NICUs, possible transposable element transmission between NICUs, and repeated importation of ESBL-KP from the community.
\end{abstract}

Keywords: ESBL-KP; dual technology WGS; neonatal ICU; outbreak

\section{Introduction}

Extended-spectrum beta-lactamase-producing Enterobacterales (ESBL-PE) are considered a "serious threat" by the US Centers for Disease Control [1] and a "critical priority" for antibiotic development by the World Health Organization [2]. A study of 890 US hospitals estimated that the incidence of infections caused by ESBL-PE increased by 53\% from 2012 to 2017 [3]. While ESBL-PE 
were initially almost exclusively nosocomial pathogens, over the last two decades the epidemiology has shifted and ESBL-PE, including K. pneumoniae, are now carried and spread in the community as well [4].

ESBL-producing K. pneumoniae (ESBL-KP) have been a noted cause of outbreaks in neonatal intensive care units (NICUs) [5-8]. Strains involved in these outbreaks classically represent transmission of a single clone, spread by the hands of healthcare workers or by fomites [9]. Such clonal outbreaks may be spread between wards and institutions by patient transfers or by healthcare workers who work at more than one site [7]. In these scenarios, a lack of clonal relatedness among infected patients or affected wards does not necessarily rule out nosocomial spread, as ESBL genes may spread by plasmids or transposable elements [8]. On the other hand, with the rise of community carriage of ESBL-producing strains, what appear to be nosocomial outbreaks in the NICU may actually represent multiple introductions from the community, either by vertical transmission during delivery or by visiting family members who are ESBL-PE carriers. In these scenarios, it is expected that the strains and plasmids will be unrelated in contrast to cases of clonal spread.

Between July and September 2017, the National Center for Antibiotic Resistance and Infection Control in the Israeli Ministry of Health was notified independently by NICUs in three hospitals in a 20-km radius of outbreaks caused by ESBL-KP. In this study, we describe the molecular investigation of the consecutive (slightly overlapping) outbreaks using a methodological approach that included typing of all isolates and whole genome sequencing (WGS) of isolates representing clonal groups. We aimed to determine whether these outbreaks represented nosocomial transmission of a single clone or plasmid between all three institutions, separate nosocomial transmission within each institution, or unrelated events of ESBL importation from the community.

\section{Results}

Forty-three isolates from 40 unique patients in the three NICUs were included in this study. Four isolates from different anatomic sites came from one patient and were not included in the epidemiological timeline. All isolates were third-generation cephalosporin-resistant K. pneumoniae that were suspected ESBL producers. An epidemiological investigation was conducted without yielding conclusive results, which prompted a molecular analysis to answer the following questions.

\subsection{Was This a Clonal Outbreak Involving All Three Hospitals?}

BOX-PCR identified a distinct dominant clone in each of the three NICUs, as well as secondary and satellite (singleton) clones (Figure 1 and Table S1). Although this finding suggests that the outbreaks were not clonally related, to further evaluate the possibility that the different clones did, in fact, share a common origin, we selected representative isolates from each clone for WGS. We found that each of the BOX-PCR clones belonged to a distinct ST-type (Figure 1). All sequence types belonged to the KpI phylogroup but were distant from each other (Figure 2). One satellite clone (Clone E, ST35) isolated from a patient in NICU-2 on 11 August 2017 was identified as the dominant clone in the outbreak in NICU-3, where it was first isolated four weeks later. The ST35 NICU-2 isolate was indistinguishable from the ST35 NICU-3 isolates by core-genome alignment: almost no dissimilarity distance was found between them (Figure S1). We believe that this represents transmission from NICU-2 to NICU-3 or a reoccurring introduction from a common reservoir. Thus, this was not a single clonal outbreak involving all three hospitals; rather, a suspected transmission event occurred between NICU-2 and NICU-3, leading to a clonal outbreak in NICU-3. 


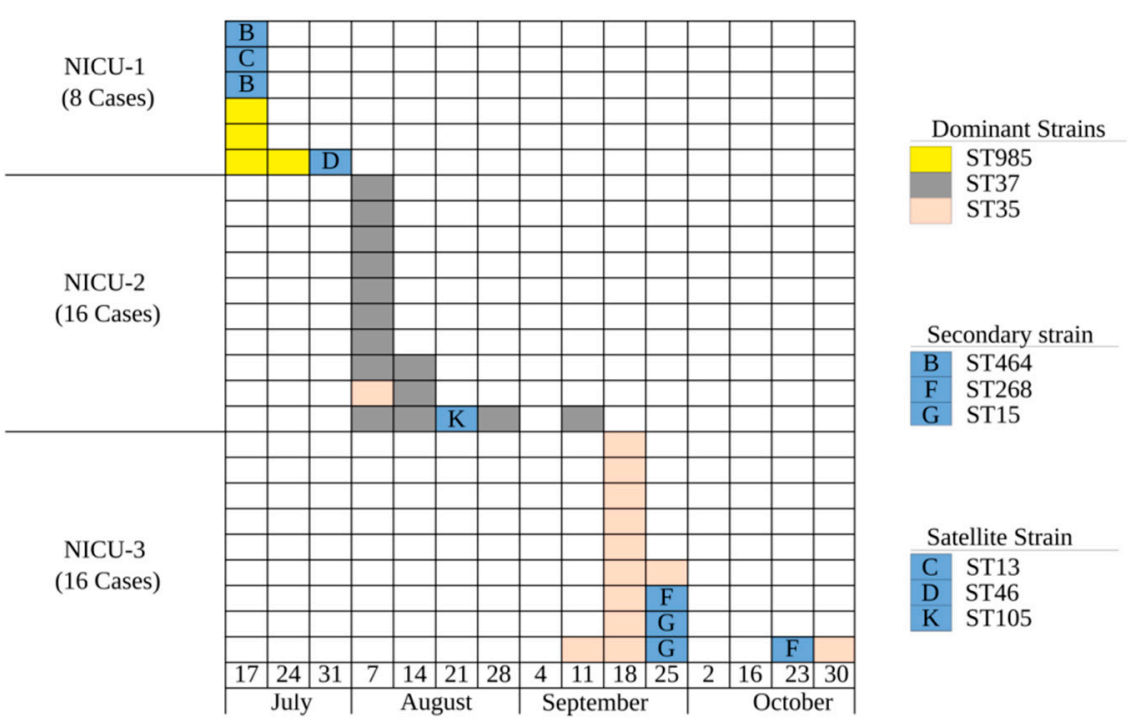

Figure 1. Epidemic curve of extended-spectrum beta-lactamase-producing Klebsiella pneumoniae (ESBL)-producing K. pneumoniae outbreaks in three neonatal intensive care units (NICUs): cases per week, 17 July-1 November 2017. Clones were determined by BOX-PCR and MLST and are color coded. Secondary strains (appeared more than once) and satellite strains (singletons) are coded by BOX-PCR letters as presented in Table S1.

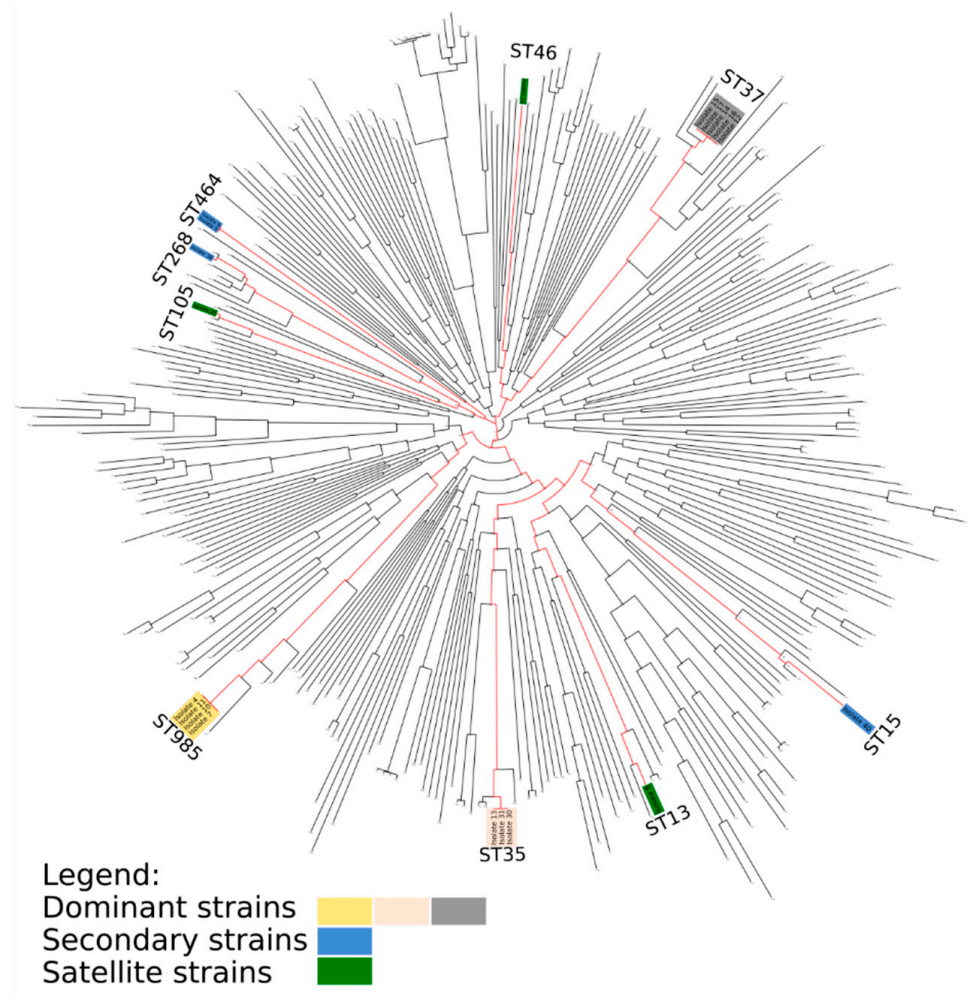

Figure 2. KpI, a major K. pneumoniae phylogenetic group, supplemented with ESBL-producing K. pneumoniae isolates from the neonatal intensive care unit outbreaks reported here. Dominant clones are in yellow, gray, and pink. Secondary and satellite strains are in blue and green, respectively.

\subsection{Were the Outbreaks a Result of Plasmid Dissemination Between Clones and NICUs?}

All three dominant clones had two similar replication loci, IncFIB and IncFII, but the pMLST of their IncFII differed (Figure S2). Only the dominant clone in NICU-2 had IncFIA, and only the dominant clone in NICU-3 had a secondary IncFIB replication locus. Complete genome sequencing 
of representative isolates revealed a single plasmid in the NICU-1-dominant clone and two different plasmids in the NICU-2- and NICU-3-dominant clones. IncFIB(K)-IncFII (in NICU-1 and NICU-3) and IncFIB (in NICU-2) had similar operon composition and carried multiple antibiotic resistance genes, but had different gene arrangements and antibiotic resistance islands (ARI) (Figure 3). All three dominant clones carried several similar plasmid operons, including iron uptake $(f e c)$, copper $(p c o)$, arsenic (ars) and silver (sil) resistance. Surprisingly, the plasmids of the NICU-2-dominant clone did not carry a $b l a_{\mathrm{CTX}-\mathrm{M}}$ gene; instead, its $b l a_{\mathrm{CTX}-\mathrm{M}-15}$ was carried chromosomally.

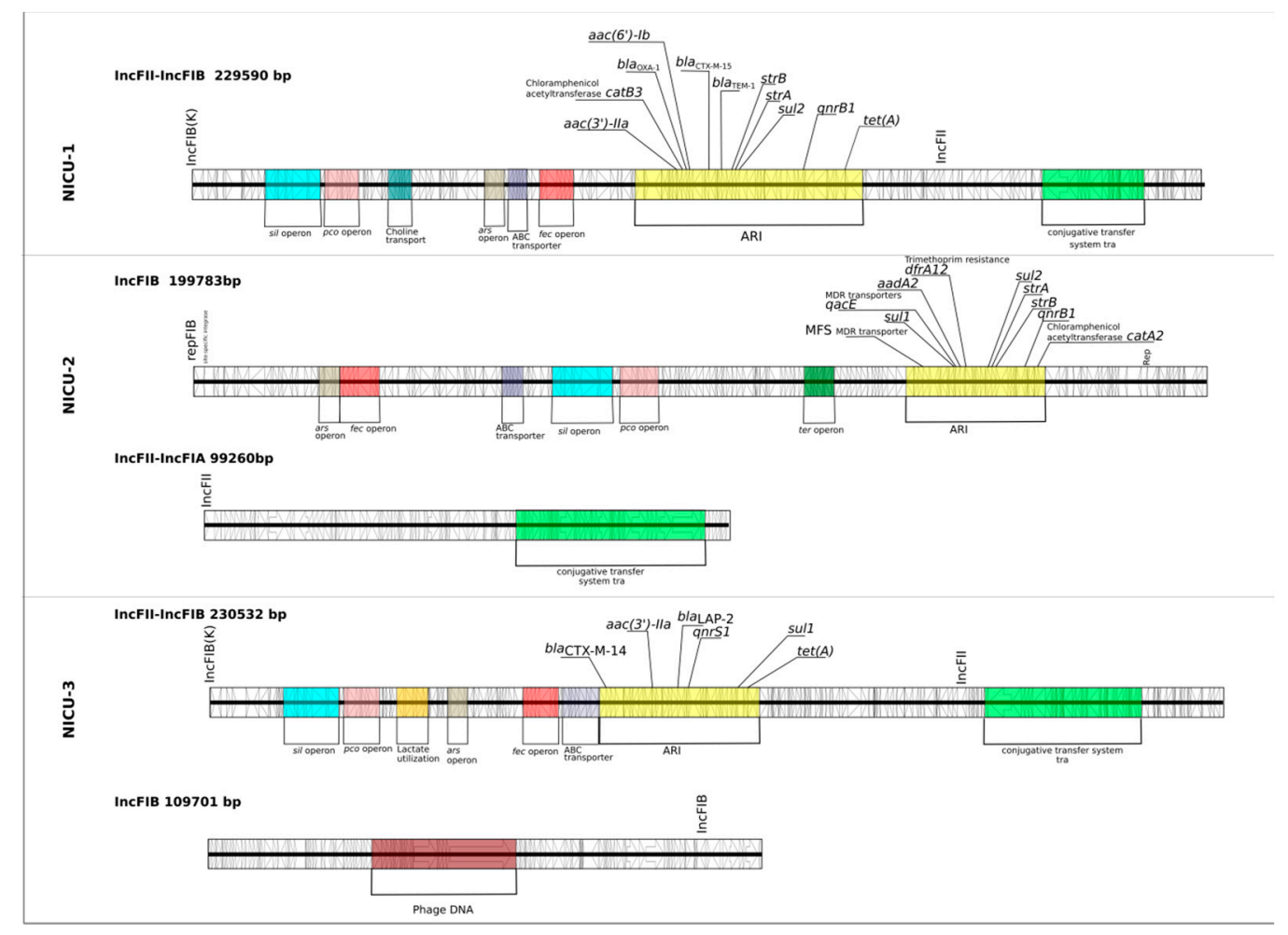

Figure 3. Schematic view of complete plasmids (over $10 \mathrm{Kbp}$ ) found by whole genome sequencing of dominant clones in outbreaks in three neonatal intensive care units. Antibiotic resistance genes and major operons are annotated.

The ST35 satellite isolate in NICU-2 had the same plasmid profile as the ST35-dominant clone in NICU-3. None of the other satellite clones shared a plasmid profile with the dominant clones. In NICU-1, one satellite isolate (isolate 11, ST46) and the dominant clone had different plasmids but shared a similar ARI $\left(b l a_{\mathrm{OXA}-1}, b l a_{\mathrm{TEM}-1}, b l a_{\mathrm{CTX}-\mathrm{M}-15}, \operatorname{str} B, \operatorname{str} A\right.$ and $\left.s u l 2\right)$. This may represent ARI dissemination, resulting in a mosaic plasmid.

In summary, we found no plasmid dissemination across clones. The NICU-2-dominant clone's

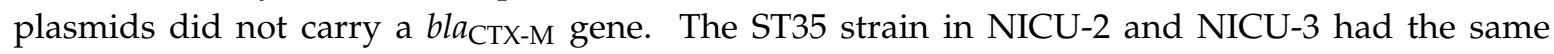
plasmid profile.

\subsection{Were the Outbreaks the Result of Dissemination of a Bla ${ }_{C T X-M}$ Transposable Element?}

The bla $a_{\text {CTX-M-15 }}$ gene was present in all NICU-1 isolates and in all NICU-2 isolates except for the ST35 isolate. All ST35 isolates carried a bla $a_{\text {CTX-M-14 }}$ gene, while the two satellite clones in NICU-3 (ST268 and ST15) carried a bla $a_{\mathrm{CTX}-\mathrm{M}-15}$ gene. The bla $a_{\mathrm{CTX}-\mathrm{M}-15}$ gene in NICU-1 was carried on an ISEcp 1 insertion sequence (IS) element. In the dominant clone, the IS was within a disrupted Tn3 transposon and flanked by antibiotic resistance genes (bla $a_{\mathrm{TEM}-1}, b l a_{\mathrm{OXA}-1}, \operatorname{str} B, \operatorname{str} A$ and $\left.\operatorname{sul} 2\right)$ while in the satellite clones, other diverse flanking elements were found (Figure S3). In the NICU-2-dominant strain, $b l a_{\text {CTX-M-15 }}$ was identified on the same ISEcp1 IS within the Tn3 transposon as in NICU-1. 
Interestingly, in the NICU-2-dominant clone, the transposome was carried on the chromosome and not

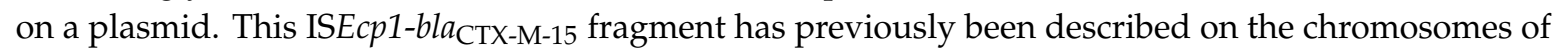
isolates belonging to several ST types [10-12]. A comparison of these fragments is illustrated in Figure 4. The ISEcp1-bla CTX-M-15 fragments from the dominant clones in NICU-1 and NICU-2 are more closely related to each other than to the fragments described in previous studies. In particular, the Tn3-like transposon is unique to the fragments from NICU-1 and NICU-2. The NICU-3-dominant clone had a $b l a_{\mathrm{CTX}-\mathrm{M}-14}$ gene adjacent to the transposable elements ISEcp1 (upstream) and IS5 (downstream).

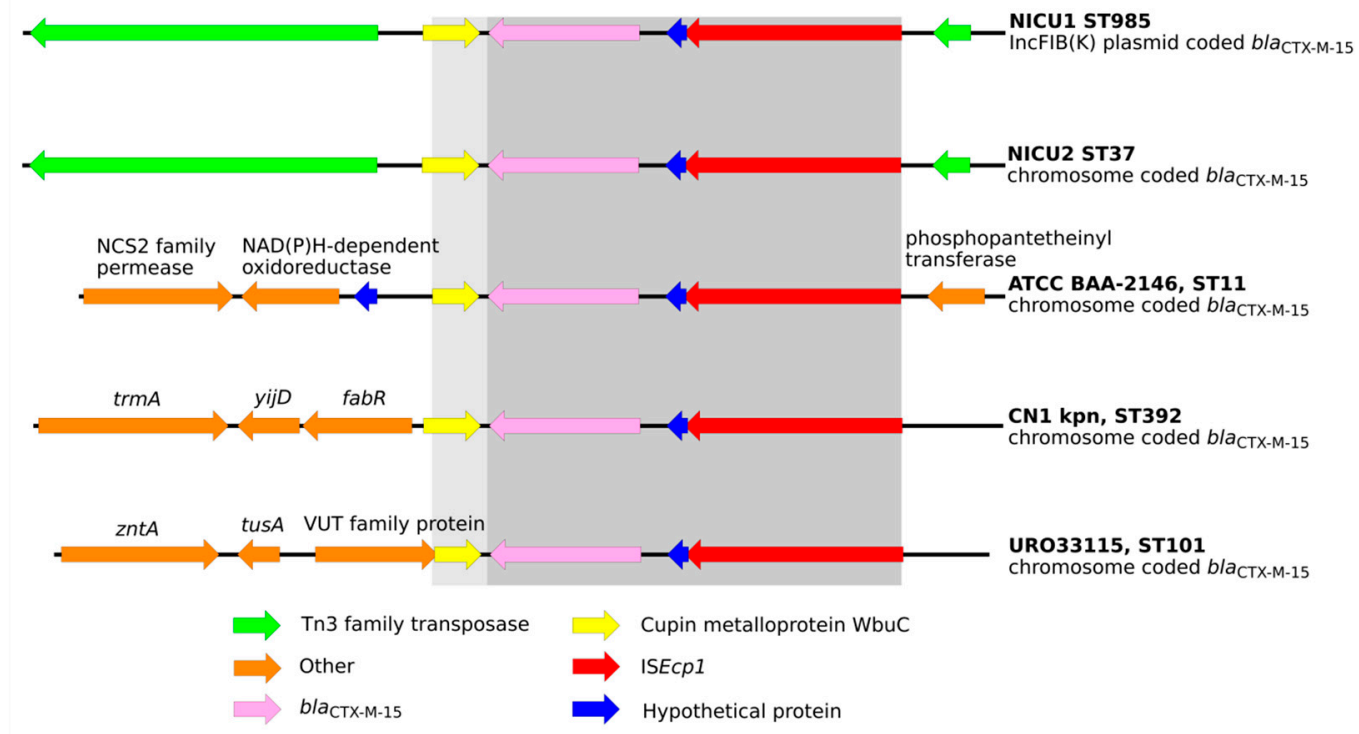

Figure 4. Schematic view of genomic fragments surrounding the ISEcp 1 transposable element containing the bla previously described K. pneumoniae isolates. Dark gray indicates 100\% similarity between all isolates and light gray indicates high similarity.

In summary, the NICU-1- and NICU-2-dominant clones had bla transposable element (Tn3-ISEcp1) but at different locations, i.e., on a plasmid in NICU-1 and on a chromosome in NICU-2. Because Tn3-ISEcp1-bla CTX-M-15 is a common genetic complex, we cannot determine whether it was transmitted from NICU-1 to NICU-2 or introduced into both independently.

\subsection{What May Have Contributed to the Success of the Dominant Clones?}

$A M R$ genes. All isolates were resistant to expanded-spectrum cephalosporins agents and were multi-drug resistant. Satellite clones generally had fewer AMR genes than the dominant clones: a mean of 9 vs. 8.6 (Table S2). Examining specific resistance genes and gene families, we found that almost all clones had QNR genes and most had a sul2 gene. The NICU-3-dominant clone had a sul1 gene and the NICU-2-dominant clone had both sul1 and sul2 genes. The NICU-1-dominant clone carried 2 AAC genes $\left(a a c\left(6^{\prime}\right)-I b\right.$ and $\left.a a c\left(3^{\prime}\right)-I I a\right)$. All study clones carried both a bla $a_{\mathrm{CTX}-\mathrm{M}}$ gene and an ESBL SHV gene. Thus, carrying genes from two ESBL families was not unique to dominant clones. In NICU-1, the dominant clone (ST985) and two satellite clones (ST13 and ST46) also carried a class D beta-lactamase, $b l a_{\mathrm{OXA}-1}$. Because $b l a_{\mathrm{OXA}-1}$ hydrolyzes broad-spectrum penicillins such as piperacillin and is not inhibited by tazobactam, it may confer selective advantage beyond ESBL carriage in settings where piperacillin/tazobactam is widely used. In sum, we found no significant difference in resistance potential between the dominant and satellite clones.

Virulence genes. Few virulence genes were identified in the study clones, but the dominant clone in NICU-1 and NICU-2 and one of the satellite strains in NICU-3 carried genes coding for yersiniabactin, a siderophore. Each clone had a different variant of yersiniabactin with different integrative conjugative 
elements (ICE) (Table S1), suggesting independent events of gene acquisition. All dominant clones carried iron uptake ( $f e c)$, copper resistance ( $p c o$ ), arsenic resistance (ars), and silver resistance (sil) genes and an $\mathrm{ABC}$ transport system.

\section{Discussion}

Our study demonstrates the complex molecular epidemiology of ESBL-producing K. pneumoniae, which are of growing concern for healthcare providers worldwide [3]. We found that each of the consecutive outbreaks in NICUs in three hospitals in a small geographic region was caused by a different dominant clone of $b l a_{\mathrm{CTX}-\mathrm{M}}$-producing $\mathrm{K}$. pneumoniae. In each NICU, along with the dominant clone, 1 to 3 secondary and satellite clones were also found. We determined that a satellite clone in NICU-2 was likely the origin of, or from a common reservoir as, the dominant clone in NICU-3, suggesting that the outbreaks did not represent completely independent nosocomial transmission within each institution. An epidemiological investigation focusing on common patients and healthcare workers between these two NICUs failed to uncover the link. We found no evidence of plasmid dissemination between clones as reported in other studies [6]. However, certain plasmids became more common because of clonal expansion of the ST35. Finally, the presence of singleton satellite clones D (in NICU-1) and K (in NICU-2) may represent ESBL importation from the community or from elsewhere in the hospital.

We found that all NICU-1 and most NICU-2 clones carried $b l a_{\mathrm{CTX}-\mathrm{M}-15}$. Interestingly, $b l a_{\mathrm{CTX}-\mathrm{M}-15}$ in NICU-1 was plasmid coded, while in the dominant clone of NICU-2 it was chromosomally coded. One NICU-2 satellite clone (ST35) carried bla $a_{\text {CTX-M-14 }}$ and not bla $a_{\mathrm{CTX}-\mathrm{M}-15}$. The NICU-3-dominant clone, which likely originated from NICU-2, also carried bla $a_{\mathrm{CTX}-\mathrm{M}-14}$, while the two satellite clones in

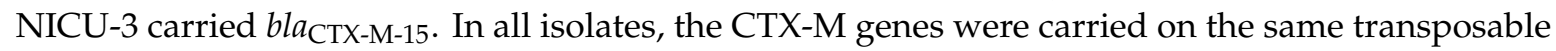
element, ISEcp1, which is a common integrative element responsible for dissemination of ESBL genes. However, the flanking regions of the transposable elements varied between clones, suggesting multiple transposition events. We did not find evidence that such events linked the outbreaks investigated here, unlike previously reported events involving other transposable elements [13] or ISEcp1 [14].

Most ST types in our study are not frequently cited as ESBL-KP-causing outbreaks, except for ST15 [14,15], which appeared only as a satellite clone, and ST-37, a frequently found carbapenemresistant strain [15]. Similarly, ESBL-KP clones responsible for other NICU outbreaks were not previously recognized as problematic nosocomial strains [6,7], which suggests that they were not part of the general hospital ecosystem.

We explored possible determinants of the dominant clones' success. The dominant clones had on average a higher number of AMR genes, but no unique resistance mechanism. The dominant clones had various mechanisms of iron acquisition, including the citrate-mediated iron uptake system (fec), the FhuDCB locus, a chromosomal ABC-dependent Fe-siderophore uptake mechanism [16], and yersiniabactin. However, some satellite clones also carried these genes, including the plasmid-coded virulence factors. Thus, we did not find a specific determinant that explained the dominance of specific clones. We believe that in an era of wide dissemination of multidrug-resistant organisms, the success of a specific clone is likely a combination of particular clone characteristics, the NICU ecosystem, infection control practices, and chance.

Our study has several limitations. Among the potentially infected patients, only those hospitalized during the investigation were screened. Furthermore, not all isolates from ESBL-KP-positive patients were subjected to sequencing and only the selected dominant clones were sequenced using both long and short read sequencing technologies. However, using WGS on selected isolates provided useful information for understanding the ESBL-KP NICU outbreaks that could not be gleaned by standard epidemiology and traditional molecular techniques. Furthermore, by merging BOX-PCR with short and long read sequencing technologies, this study presented in-depth genomic analysis but at a lower cost. This approach may encourage smaller epidemiological laboratories to use genomic tools for epidemiological studies. 


\section{Materials and Methods}

\subsection{Patients and Setting}

Three NICUs, within $20 \mathrm{~km}$ from each other, of different sizes were investigated in this study; NICUs 1 and 2 were of similar sizes with 16 and 24 beds, respectively. NICU-3 had 49 beds at the time of the outbreak. In all three hospitals, an index case of a neonate with bacteremia with an ESBL-producing Enterobacteriaceae isolated prompted screening of all exposed neonates. Identified carriers were placed in cohorts with isolation precautions and bacterial samples were sent to the reference laboratory at the National Center for Antibiotic Resistance and Infection Control.

\subsection{Isolates and Molecular Analysis}

Isolates were grown on MacConkey agar (Hy Laboratories Ltd., Rehovot, Israel) and identified to the species level by VITEK ${ }^{\circledR}$ MS (bioMérieux SA, Marcy l'Etoile, France). Antibiotic susceptibility was determined by the VITEK ${ }^{\circledR} 2$ system. Confirmatory testing for ESBL production was carried out by double-disc diffusion according to CLSI guidelines. Isolates were stored at $-80{ }^{\circ} \mathrm{C}$ and sub-cultured before testing. Clonality was determined using BOX-PCR [17]. Representative isolates from each BOX-PCR type and several replicates from the dominant clones were subjected to genomic analysis, to further understand the outbreaks' genetic context. Only one isolate from the patient with multiple isolates was sent for sequencing. Isolate DNA was sent to the Sequencing Core at the University of Illinois at Chicago. Sequencing libraries were prepared using the Nextera XT kit (Illumina Inc., San Diego, CA, USA) and sequenced on the Illumina NextSeq 500 using the high output $2 \times 150$ bp kit (Illumina Inc.). Each genome was sequenced to an average sequence depth of approximately $0.8 \mathrm{~Gb}$ per genome.

Quality validation of sequence data, adapter trimming, and ambiguous base removal were done by Fastp (v0.19.5) [18]. Next, samples were assembled using SPAdes (v3.12.0) [19] under default conditions. Gene calling and annotation were achieved using Prokka (v1.13.3) software [20]. One representative isolate (the isolate with the best N50 assembly score) from each NICU's dominant clone was also sequenced using long read sequencing technology. Libraries were prepared using the multiplexing kit Rapid Barcoding Sequencing (SQK-RBK004) and sequenced on a MinION device (Oxford Nanopore Technologies (ONT), Oxford, UK) according to the manufacturer's instructions. Sequence assembly and de-multiplexing were done using Guppy software (v3.2.2) (ONT). Illumina reads were quality screened using Fastp and then assembled together with ONT reads using Unicycler (v0.4.8) [21]. Annotation and downstream application were performed as described for the genomes sequenced only by Illumina technology.

\subsection{Phylogenomics, Resistomes, and Virulence Factors}

A list (Table S3) of representative genomes of most KpI MLSTs [22] was downloaded from the SRA and PATRIC databases and was assembled and annotated in the same way as was done for this study's isolates. All genomes (downloaded and new) were subjected to pan-genome analysis using Roary software [23]. Core genomes were aligned using default settings of the MAFFT (v7.407) alignment algorithm [24]. The resulting multi-gene alignment was subject to phylogenetic reconstruction using FastTree (v2.1.11) [25]. The resulting KpI tree was used to determine the phylogenetic relationship between clones in light of the greater $\mathrm{KpI}$ group in order to determine whether all clonal groups were distinct. The same strategy was employed for K. pneumoniae ST35 genomes listed in Figure S1. We used Kleborate (v0.3.0) (https://github.com/katholt/Kleborate) to identify and classify antibiotic resistance genes and MLST types and to predict the O-antigen and K-locus types.

\subsection{Plasmid Content Analysis}

Plasmid prediction was conducted by searching genome sequences against the PlasmidFinder database [26]. Plasmid MLST (pMLST) was assigned using the PubMLST web interface 
(https://pubmlst.org/plasmid/). For the genomes that were sequenced using long read technology and resulted in circular chromosomes and plasmids, the plasmid content was annotated and plotted using EasyFig v2.2.2 [27]. DNA fragments (5300-5800bp) from all sequenced isolates, with a transposable element carrying the $b a_{\mathrm{CTX}-\mathrm{M}-15}$ gene were retrieved and plotted using EasyFig. These DNA sequences were compared to similar DNA fragments from genomics data previously published for K. pneumoniae strain ATCC BAA-2146 (Kpn2146) [10], CN1 [11] and UR033115 [12]. Contigs carrying bla CTX-M-15 were compared in a similar manner in order to examine the $\beta$-lactamase flanking regions.

All sequence data produced in this study were submitted to the sequence read archive (SRA) under bioproject accession number PRJNA529587 (Table S3).

\section{Conclusions}

The investigation of three overlapping, consecutive ESBL-KP NICU outbreaks in nearby hospitals has shed light on their epidemiological complexity and the need to support investigations by WGS. We found that each outbreak had a dominant clone. Thus, even in the setting of high endemicity, a cluster of ESBL-KP should be treated as an outbreak and appropriately investigated.

Supplementary Materials: The following are available online at http://www.mdpi.com/2079-6382/9/10/705/s1. Table S1. List of isolates and their accession numbers, BOX-PCR clade and antibiogram along with assembly statistic, dates, and clinical source. Table S2. Genetic characteristics of genomes found in outbreaks of ESBL-producing K. pneumoniae in three neonatal intensive care units. Table S3. List of KpI reference genomes used to build the KpI phylogenetic tree. Figure S1. Core-genome alignment phylogenetic tree of ST35 genomes from NICU-2 and NICU-3 isolates together with 21 ST35 genomes from the SRA database. ST35 genomes from NICU-2 and NICU-3 isolates are monophyletic and have almost no dissimilarity distance. Figure S2. Plasmid replication control loci presence and absence according to the PlasmidFinder database and pMLST typing based on PubMLST. The asterisks indicate the dominant clone in each neonatal intensive care unit (NICU). Figure S3. ISEcp1-blaCTX-M-15 elements from different isolates show different flanking regions. AR genes are marked according to the legend.

Author Contributions: Conceptualization: S.F., N.R. and Y.C. Investigation: V.S., R.C., B.S.K., E.S., S.C., S.S. and Y.C. Project administration: M.J.S. Writing of original draft: S.F. and N.R. Revisions and editing: E.T. and Y.C. All authors have read and agreed to the published version of the manuscript.

Funding: This research received no external funding.

Conflicts of Interest: Authors do not have an association that might pose a conflict of interest.

\section{References}

1. Centers for Disease Control and Prevention (United States). Antibiotic/Antimicrobial Resistance. Biggest Threats and Data. Available online: https://www.cdc.gov/drugresistance/biggest-threats.html? CDC_AA_refVal=https $\% 3 \mathrm{~A} \% 2 \mathrm{~F} \% 2 \mathrm{Fwww.cdc} . g o v \% 2 \mathrm{Fdrugresistance} \% 2 \mathrm{Fbiggest}$ threats.html\#groupb (accessed on 1 August 2020).

2. World Health Organization. Prioritization of Pathogens to Guide Discovery, Research and Development of New Antibiotics for Drug-Resistant Bacterial Infections, including Tuberculosis; World Health Organization: Geneva, Switzerland, 2017.

3. Jernigan, J.A.; Hatfield, K.M.; Wolford, H.; Nelson, R.E.; Olubajo, B.; Reddy, S.C.; McCarthy, N.; Paul, P.; McDonald, L.C.; Kallen, A.; et al. Multidrug-resistant bacterial infections in U.S. hospitalized patients, 2012-2017. N. Engl. J. Med. 2020, 382, 1309-1319. [CrossRef] [PubMed]

4. Bush, K.; Bradford, P.A. Epidemiology of $\beta$-lactamase-producing pathogens. Clin. Microbiol. Rev. 2020, 33, 00047-19. [CrossRef] [PubMed]

5. Haller, S.; Eller, C.; Hermes, J.; Kaase, M.; Steglich, M.; Radonić, A.; Dabrowski, P.W.; Nitsche, A.; Pfeifer, Y.; Werner, G.; et al. What caused the outbreak of ESBL-producing Klebsiella pneumoniae in a neonatal intensive care unit, Germany 2009 to 2012? Reconstructing transmission with epidemiological analysis and whole-genome sequencing. BMJ Open 2015, 5, e007397. [CrossRef] [PubMed]

6. Peltier, F.; Choquet, M.; Decroix, V.; Adjidé, C.C.; Castelain, S.; Guiheneuf, R.; Pluquet, E. Characterization of a multidrug-resistant Klebsiella pneumoniae ST607-K25 clone responsible for a nosocomial outbreak in a neonatal intensive care unit. J. Med. Microbiol. 2019, 68, 67-76. [CrossRef] [PubMed] 
7. Wang, Y.; Luo, C.; Du, P.; Hu, J.; Zhao, X.; Mo, D.; Du, X.; Xu, X.; Li, M.; Lu, H.; et al. Genomic epidemiology of an outbreak of Klebsiella pneumoniae ST471 producing extended-spectrum $\beta$-Lactamases in a neonatal intensive care unit. Infect. Drug Resist. 2020, 13, 1081-1090. [CrossRef] [PubMed]

8. Stapleton, P.J.M.; Murphy, M.; McCallion, N.; Brennan, M.; Cunney, R.; Drew, R.J. Outbreaks of extended spectrum beta-lactamase-producing Enterobacteriaceae in neonatal intensive care units: A systematic review. Arch. Dis. Child. Fetal Neonatal Ed. 2015, 101, 72-78. [CrossRef] [PubMed]

9. Paterson, D.L.; Bonomo, R.A. Extended-spectrum $\beta$-Lactamases: A clinical update. Clin. Microbiol. Rev. 2005, 18, 657-686. [CrossRef]

10. Hudson, C.M.; Bent, Z.W.; Meagher, R.J.; Williams, K.P. Resistance determinants and mobile genetic elements of an NDM-1-Encoding Klebsiella pneumoniae Strain. PLoS ONE 2014, 9, e99209. [CrossRef]

11. Huang, W.; Wang, G.; Sebra, R.; Zhuge, J.; Yin, C.; Aguero-Rosenfeld, M.E.; Schuetz, A.N.; Dimitrova, N.; Fallon, J.T. Emergence and evolution of multidrug-resistant Klebsiella pneumoniae with both blaKPC and blaCTX-M integrated in the chromosome. Antimicrob. Agents Chemother. 2017, 61, e00076-17. [CrossRef]

12. Mshana, S.E.; Fritzenwanker, M.; Falgenhauer, L.; Domann, E.; Hain, T.; Chakraborty, T.; Imirzalioglu, C. Molecular epidemiology and characterization of an outbreak causing Klebsiella pneumoniae clone carrying chromosomally located bla CTX-M-15 at a German University-Hospital. BMC Microbiol. 2015, 15, 122. [CrossRef]

13. Sheppard, A.E.; Stoesser, N.; Wilson, D.J.; Sebra, R.; Kasarskis, A.; Anson, L.W.; Giess, A.; Pankhurst, L.J.; Vaughan, A.; Grim, C.J.; et al. Nested Russian doll-like genetic mobility drives rapid dissemination of the Carbapenem resistance GeneblaKPC. Antimicrob. Agents Chemother. 2016, 60, 3767-3778. [CrossRef] [PubMed]

14. Perdigão, J.; Modesto, A.; Pereira, A.L.; Neto, O.; Matos, V.; Godinho, A.; Phelan, J.; Charleston, J.; Spadar, A.; De Sessions, P.F.; et al. Whole-genome sequencing resolves a polyclonal outbreak by extended-spectrum beta-lactam and carbapenem-resistant Klebsiella pneumoniae in a Portuguese tertiary-care hospital. Microb. Genom. 2020. [CrossRef]

15. Wyres, K.L.; Lam, M.M.C.; Holt, K.E. Population genomics of Klebsiella pneumoniae. Nat. Rev. Genet. 2020, 18, 344-359. [CrossRef] [PubMed]

16. Miethke, M.; Marahiel, M.A. Siderophore-based iron acquisition and pathogen control. Microbiol. Mol. Biol. Rev. 2007, 71, 413-451. [CrossRef]

17. Rakovitsky, N.; Frenk, S.; Kon, H.; Schwartz, D.; Temkin, E.; Solter, E.; Paikin, S.; Cohen, R.; Schwaber, M.J.; Carmeli, Y; et al. Fourier transform infrared spectroscopy is a new option for outbreak investigation: A retrospective analysis of an extended-spectrum-beta-lactamase-producing Klebsiella pneumoniae outbreak in a neonatal intensive care unit. J. Clin. Microbiol. 2020, 58, 00098-20. [CrossRef] [PubMed]

18. Chen, S.; Zhou, Y.; Chen, Y.; Gu, J. fastp: An ultra-fast all-in-one FASTQ preprocessor. Bioinformatics 2018, 34, i884-i890. [CrossRef] [PubMed]

19. Bankevich, A.; Nurk, S.; Antipov, D.; Gurevich, A.A.; Dvorkin, M.; Kulikov, A.S.; Lesin, V.M.; Nikolenko, S.I.; Pham, S.; Prjibelski, A.D.; et al. SPAdes: A new genome assembly algorithm and its applications to single-cell sequencing. J. Comput. Biol. 2012, 19, 455-477. [CrossRef] [PubMed]

20. Seemann, T. Prokka: Rapid prokaryotic genome annotation. Bioinformatics 2014, 30, 2068-2069. [CrossRef]

21. Wick, R.R.; Judd, L.M.; Gorrie, C.; Holt, K.E. Unicycler: Resolving bacterial genome assemblies from short and long sequencing reads. PLoS Comput. Biol. 2017, 13, e1005595. [CrossRef]

22. Holt, K.E.; Wertheim, H.; Zadoksm, R.N.; Baker, S.; Whitehouse, C.A.; Dance, D.; Jenney, A.; Connor, T.R.; Hsu, L.Y.; Severin, J.; et al. Genomic analysis of diversity, population structure, virulence, and antimicrobial resistance in Klebsiella pneumoniae, an urgent threat to public health. Proc. Natl. Acad. Sci. USA 2015, 112, E3574-E3581. [CrossRef]

23. Page, A.J.; Cummins, C.A.; Hunt, M.; Wong, V.K.; Reuter, S.; Holden, M.T.; Fookes, M.; Falush, D.; Keane, J.A.; Parkhill, J. Roary: Rapid large-scale prokaryote pan genome analysis. Bioinformatics 2015, 31, 3691-3693. [CrossRef] [PubMed]

24. Katoh, K.; Standley, D.M. MAFFT multiple sequence alignment software version 7: Improvements in performance and usability. Mol. Biol. Evol. 2013, 30, 772-780. [CrossRef]

25. Price, M.N.; Dehal, P.S.; Arkin, A.P. FastTree 2-Approximately maximum-likelihood trees for large alignments. PLoS ONE 2010, 5, e9490. [CrossRef] 
26. Carattoli, A.; Zankari, E.; García-Fernández, A.; Larsen, M.V.; Lund, O.; Villa, L.; Aarestrup, F.M.; Hasman, H. In SilicoDetection and typing of plasmids using PlasmidFinder and plasmid multilocus sequence typing. Antimicrob. Agents Chemother. 2014, 58, 3895-3903. [CrossRef] [PubMed]

27. Sullivan, M.J.; Petty, N.K.; Beatson, S.A. Easyfig: A genome comparison visualizer. Bioinformatics 2011, 27, 1009-1010. [CrossRef] [PubMed]

Publisher's Note: MDPI stays neutral with regard to jurisdictional claims in published maps and institutional affiliations.

(C) 2020 by the authors. Licensee MDPI, Basel, Switzerland. This article is an open access article distributed under the terms and conditions of the Creative Commons Attribution (CC BY) license (http://creativecommons.org/licenses/by/4.0/). 